\title{
Study of leptonic and semileptonic kaon decays at CERN
}

\author{
Riccardo Fantechi ${ }^{* \dagger}$ \\ INFN - Sezione di Pisa and CERN \\ E-mail: fantechi@cern.ch
}

\begin{abstract}
During 2003 and 2004, the NA48/2 experiment has collected at the CERN SPS the world largest amount of charged kaon decays, with the main goal to search for direct $\mathrm{CP}$ violation in the decay of $\mathrm{K}^{ \pm}$into three pions. From a dedicated data sample collected in 2004, the analysis of $K_{e 3}^{ \pm}$and $K_{\mu 3}^{ \pm}$decay data has allowed precise measurements of the form factors in various parametrizations. Later, in 2007-2008, using the same detector but a modified beam and trigger logic, the NA62 experiment has taken data to measure the ratio of $K \rightarrow e v$ and $K \rightarrow v$ leptonic decays and has obtained a sub-percent measurement of the ratio $R_{K}=\Gamma\left(K_{e 2}\right) / \Gamma\left(K_{\mu 2}\right)$.
\end{abstract}

The European Physical Society Conference on High Energy Physics -EPS-HEP2013

18-24 July 2013

Stockholm, Sweden

\footnotetext{
* Speaker.

for the NA48/2 and NA62 Collaborations: F. Ambrosino, A. Antonelli, G. Anzivino, R. Arcidiacono, W. Baldini, S. Balev, J.R. Batley, M. Behler, S. Bifani, C. Biino, A. Bizzeti, B. Bloch-Devaux, G. Bocquet, V. Bolotov, F. Bucci, N. Cabibbo, M. Calvetti, N. Cartiglia, A. Ceccucci, P. Cenci, C. Cerri, C. Cheshkov, J.B. Chèze, M. Clemencic, G. Collazuol, F. Costantini, A. Cotta Ramusino, D. Coward, D. Cundy, A. Dabrowski, G. D’Agostini, P. Dalpiaz, C. Damiani, H. Danielsson, M. De Beer, G. Dellacasa, J. Derré, H. Dibon, D. Di Filippo, L. DiLella, N. Doble, V. Duk, J. Engelfried, K. Eppard, V. Falaleev, R. Fantechi, M. Fidecaro, L. Fiorini, M. Fiorini, T. Fonseca Martin, P.L. Frabetti, A. Fucci, S. Gallorini, L. Gatignon, E. Gersabeck, A. Gianoli, S. Giudici, A. Gonidec, E. Goudzovski, S. Goy Lopez, E. Gushchin, B. Hallgren, M. Hita-Hochgesand, M. Holder, P. Hristov, E. Iacopini, E. Imbergamo, M. Jeitler, G. Kalmus, V. Kekelidze, K. Kleinknecht, V. Kozhuharov, W. Kubischta, V. Kurshetsov, G. Lamanna, C. Lazzeroni, M. Lenti, E. Leonardi, L. Litov, D. Madigozhin, A. Maier, I. Mannelli, F. Marchetto, G. Marel, M. Markytan, P. Marouelli, M. Martini, L. Masetti, P. Massarotti, E. Mazzucato, A. Michetti, I. Mikulec, M. Misheva, N. Molokanova, E. Monnier, U. Moosbrugger, C. Morales Morales, M. Moulson, S. Movchan, D.J. Munday, M. Napolitano, A. Nappi, G. Neuhofer, A. Norton, T. Numao, V. Obraztsov, V. Palladino, M. Patel, M. Pepe, A. Peters, F. Petrucci, M.C. Petrucci, B. Peyaud, R. Piandani, M. Piccini, G. Pierazzini, I. Polenkevich, I. Popov, Yu. Potrebenikov, M. Raggi, B. Renk, F. Retière, P. Riedler, A. Romano, P. Rubin, G. Ruggiero, A. Salamon, G. Saracino, M. Savrié, M. Scarpa, V. Semenov, A. Sergi, M. Serra, M. Shieh, S. Shkarovskiy, M.W. Slater, M. Sozzi, T. Spadaro, S. Stoynev, E. Swallow, M. Szleper, M. ValdataNappi, P. Valente, B. Vallage, M. Velasco, M. Veltri, S. Venditti, M. Wache, H. Wahl, A. Walker, R. Wanke, L. Widhalm, A. Winhart, R. Winston, M.D. Wood, S.A. Wotton, O. Yushchenko, A. Zinchenko, M. Ziolkowski.
} 


\section{Introduction}

The ratio of kaon leptonic decay rates $R_{K}=\Gamma\left(K_{e 2}\right) / \Gamma\left(K_{\mu 2}\right)$ has been computed in the Standard Model (SM) with a very good precision [1], thanks to cancellation of hadronic effects:

$$
R_{K}^{S M}=\left(m_{e} / m_{\mu}\right)^{2}\left(\frac{m_{K}^{2}-m_{e}^{2}}{m_{K}^{2}-m_{\mu}^{2}}\right)^{2}\left(1+\delta R_{Q E D}\right)=(2.477 \pm 0.001) \times 10^{-5},
$$

where $\delta R_{Q E D}=(-3.79 \pm 0.04) \%$ is a correction due to IB processes included in the definition of $R_{K}$. The ratio, helicity suppressed due to the $\mathrm{V}$-A structure of the charged weak current, is sensitive to non-SM effects. In particular MSSM allows non-vanishing $e-\tau$ mixing, mediated by $H^{+}$, which can lead to few percent enhancement of $R_{K}$ [2,3]. Measurements of $R_{K}$ were performed in the 1970s [4, 5, 6], and recently more precisely from KLOE [7] and from NA62 [8]. We report here the final measurement based on the data collected in 2007 and in 2008 by the NA62 experiment [9] and superseding the earlier NA62 result.

Semileptonic kaon decays provide the theoretically cleanest way to measure the CKM $\left|V_{u s}\right|$ parameter [10] and to perform the most accurate test of the CKM matrix unitarity. Departure of CKM unitarity in these decays will be a signal of new physics, giving hints of different dynamics in the W-quark coupling. Moreover, lepton flavour universality could be tested giving constraints on new physics. The hadronic matrix element of the semileptonic kaon decays is described in terms of two dimensionless form factors $f_{ \pm}(t)$, where $t$ is the squared four momentum transferred to the lepton system $t=\left(p_{K}-p_{\pi}\right)^{2} . f_{+}$is related to the vector exchange $\left(1^{-}\right)$to the lepton system and the $f_{0}$, describing the scalar $\left(0^{+}\right)$exchange, is defined as

$$
\left.f_{0}(t)=f_{+}(t)\right)+\frac{t}{m_{K}^{2}-m_{\pi}^{2}} f_{-}(t) .
$$

Normalized form factors $\bar{f}_{+}$and $\bar{f}_{0}$ are considered, as the vector form factor at 0 momentum cannot be directly measured. Several parametrizations exist for the form factors. We will refer here to a quadratic Taylor expansion and a pole parametrization. In the first model, the normalized form factors are function of the slope and curvature parameters $\lambda_{+, 0}$ and $\lambda_{+, 0}{ }^{\prime}$ :

$$
\bar{f}_{+, 0}(t)=1+\lambda_{+, 0}^{\prime} \frac{t}{m_{\pi}^{2}}+\frac{1}{2} \lambda_{+, 0}^{\prime}\left(\frac{t}{m_{\pi}^{2}}\right)^{2} .
$$

This type of parametrization has the disadvantge of a strong correlation between the parameters as well as the absence of their physical. In the pole parametrization, physics constraints are applied, with the introduction of pole masses of a single resonance:

$$
\bar{f}_{+}(t)=\frac{M_{V}^{2}}{M_{V}^{2}-t} \quad \bar{f}_{0}(t)=\frac{M_{S}^{2}}{M_{S}^{2}-t}
$$

\section{The NA48/2 and NA62 beams}

The beam line used in the NA48/2 [11] data taking was specifically designed to simultaneously transport positive and negative particles. The positive and negative beams were produced with 400 $\mathrm{GeV} / c$ primary protons from the SPS accelerator on a beryllium target at zero incidence angle. A central momentum and momentum bite of $60 \pm 3 \mathrm{GeV} / c$ was selected symmetrically for both positively and negatively charged particles by their passage through achromat systems, namely two 
vertical deflection magnets with opposite-sign field and a pair of dump collimators with variable but similar openings for each of the two beams, followed by two further vertical deflection magnets returning the beams onto a common axis. The beams were selected with the same geometrical acceptance and directed along a common line. Both beams were focused to similar and small spot sizes $(\approx 5 \mathrm{~mm}$ r.m.s. $)$ at the spectrometer position, so as to minimize the transverse scale of any structure within the beam spots. To collect the data used in the semileptonic decays analysis described here, the intensity of the beam was lowered and its momentum spread reduced. The beam line and setup of the earlier NA48/2 experiment were used for the NA62 data taking in 2007 [8]. The momentum of the unseparated secondary positive and/or negative hadronic beams was chosen to be $74 \mathrm{GeV} / c$ with a spread of $\pm 1.4 \mathrm{GeV} / c$ (rms). The reduction of the momentum spread was achieved with a tighter collimation scheme, partially compensated by an increase in the proton beam intensity. The configuration of the muon scrapers was changed, with the side effect of a charge-asymmetric muon halo background. For this reason, most of the data taking was recorded with the $\mathrm{K}^{+}$beam only.

\section{The NA48/2 and NA62 detector}

The momenta of charged decay products were measured by a magnetic spectrometer, housed in a tank filled with helium at nearly atmospheric pressure and placed after the decay volume. The spectrometer accomodated four drift chambers composed each of 8 planes of sense wires, and a dipole magnet located between the second and the third DCHs, which gave a horizontal transverse momentum kick to charged particles of respectively $120 \mathrm{MeV} / c$ in 2004 and $265 \mathrm{MeV} / c$ in 2007 . The corresponding measured spectrometer momentum resolution were $\sigma_{p} / p=1.02 \% \oplus 0.044 \% p$ in 2004 and $\sigma_{p} / p=0.48 \% \oplus 0.009 \% p$ in 2007, where the momentum $p$ is expressed in GeV/c. A counter hodoscope consisting of two planes of orthogonal plastic scintillator strips producing fast trigger signals was placed after the spectrometer. A $127 \mathrm{~cm}\left(27 X_{0}\right)$ thick liquid krypton electromagnetic calorimeter located further downstream is used to measure energy and position of photons and electrons. Its 13248 readout cells had a transverse size of $2 \times 2 \mathrm{~cm}^{2}$ each without longitudinal segmentation. The energy resolution was $\sigma_{E} / E=3.2 \% / \sqrt{E} \oplus 9 \% / E \oplus 0.42 \%$ (E in GeV). The spatial resolution for the transverse coordinates $\mathrm{x}$ and $\mathrm{y}$ of an isolated electromagnetic shower was $\sigma_{x}=\sigma_{y}=0.42 / \sqrt{E} \oplus 0.06 \mathrm{~cm}(\mathrm{E}$ in $\mathrm{GeV})$. A description of the detector can be found in [12].

\section{Lepton flavour violation}

The measurement of $R_{K}$ is based on data collected in the 2007 and 2008 runs. $K_{e 2}$ and $K_{\mu 2}$ events are collected simultaneously and counted, such that the final result doesn't rely on the kaon flux measurement. Moreover, several systematic effects, like reconstruction and trigger efficiencies and time dependent effects, cancel at first order. Since backgrounds and acceptance depend on momentum, $R_{K}$ is measured in 10 bins of lepton momentum between 13 and $65 \mathrm{GeV} / c$ and the results statistically combined. The ratio $R_{K}$ is computed as

$$
R_{K}=\frac{1}{D} \cdot \frac{N\left(K_{e 2}\right)-N_{B}\left(K_{e 2}\right)}{N\left(K_{\mu 2}\right)-N_{B}\left(K_{\mu 2}\right)} \cdot \frac{A\left(K_{\mu 2}\right)}{A\left(K_{e 2}\right)} \cdot \frac{f_{\mu} \cdot \mathcal{E}\left(K_{\mu 2}\right)}{f_{e} \cdot \mathcal{E}\left(K_{e 2}\right)} \cdot \frac{1}{f_{L K r}}
$$


where $N\left(K_{l 2}\right)$ are the number of selected $K_{l 2}$ candidates $(l=e, \mu), N_{B}\left(K_{l 2}\right)$ are the numbers of background events, $A\left(K_{l 2}\right)$ are the geometrical acceptance corrections, $f_{l}$ the particle identification (PID) efficiencies, $\varepsilon\left(K_{l 2}\right)$ the trigger efficiencies, $f_{L K r}$ is the global efficiency of the calorimeter readout and $\mathrm{D}=150$ the downscaling factor of the $K_{\mu 2}$ trigger. Acceptance corrections and the geometric parts of the acceptance for background processes are computed with a detailed Monte Carlo simulation, while PID, trigger and readout efficiencies are measured directly from the data.

A common selection profits from the topological similarity of the two decay modes: only one charged reconstructed track with $13<p<65 \mathrm{GeV} / c$ and likely to come from a beam kaon decay with impacts points within the acceptance of the various detectors, no $\mathrm{LKr}$ energy larger than 2 $\mathrm{GeV}$ not associated to the track (to suppress background from other decays), a maximum $3 \mathrm{~cm}$ distance between the charged track and the nominal kaon beam axis and a reconstructed decay vertex longitudinal position compatible with the nominal decay volume.

It is possible to identify kinematically the $K_{l 2}$ decays, requiring the $M_{m i s s}^{2}(l)$ to be within a window centered on zero with a width variable for different momenta, depending on the $M_{\text {miss }}^{2}(l)$ resolution. The separation of the two decays based on kinematics only is valid up to about $35 \mathrm{GeV} / c$. Particle identification is based on the ratio $E / p$ of the track energy deposit in the LKr calorimeter to its momentum measured in the spectrometer. A cut $0.95(0.9$ for $p<25 \mathrm{GeV} / c)<E / p<1.1$ selects electrons/positrons, while $E / p<0.85$ selects muons. At high momentum the major source of background is the $K_{\mu 2}$ decay with a mis-identified muon $(E / p>0.95)$, mainly due to the catastrophic bremsstrahlung in or in front of the LKr calorimeter, with a large energy deposit in it. The probability of such mis-identification has been measured as a function of momentum, preparing a positron-free muon sample by covering about $20 \%$ of the front surface of the calorimeter with 9.2 $X_{0}$ of lead placed at about $1.2 \mathrm{~m}$ of it. $55 \%$ of the kaon flux in 2007 was collected in this configuration. A detailed Geant4-based simulation of this setup has been performed to evaluate corrections to the measured probability due to ionization energy losses and bremsstrahlung in the lead. The data has been therefore divided into four samples: $K^{+}$(nolead), $K^{-}$(nolead), $K^{+}$(lead), $K^{-}$(lead).

The largest $K_{\mu 2}$ contamination in the four samples integrated over the lepton momentum is $(6.11 \pm 0.22) \%$. The contributions to its error come from: a) the limited data sample size used for the measurement, b) the uncertainty on the MC correction and c) the model dependence of the correction for the correlation between $M_{\text {miss }}^{2}(e)$ and $E / p$.

The contribution from the structure dependent (SD+) process $K \rightarrow e v \gamma$, with the gamma lost, has been estimated using a recent measurement from KLOE. Beam halo contribution to the background has been measured from the data, reconstructing $K_{e 2}$ candidates from a control $K^{-}$sample taken with the positive kaon beam blocked. In the $K_{\mu 2}$ channel, beam halo is the only significant background.

A total number of $145958 K_{e 2}$ and $42.8 \cdot 10^{7} K_{\mu 2}$ events pass all selections. The $M_{m i s s}^{2}(e)$ and $M_{\text {miss }}^{2}(\mu)$ distribution are shown respectively in Figure 1(a) and 1(b) and the values for $R_{K}$ as a function of momentum is shown in Figure 1(c). A $\chi^{2}$ fit is then performed performed over the 40 measurements (10 lepton momentum bins and four data taking conditions) as a function of momentum, taking into account the bin-to-bin correlations of the common systematic errors. The fit result [9] (with a $\chi^{2} / n d f=47 / 39$ ) is

$$
R_{K}=\left(2.488 \pm 0.007_{\text {stat. }} \pm 0.007_{\text {syst. }}\right) \cdot 10^{-5}=(2.488 \pm 0.010) \cdot 10^{-5}[9]
$$


(a)

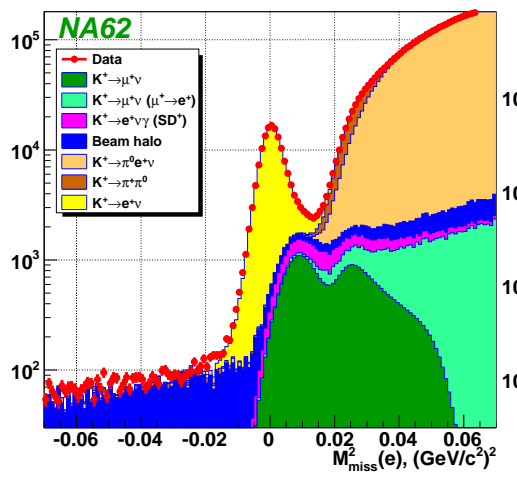

(b)

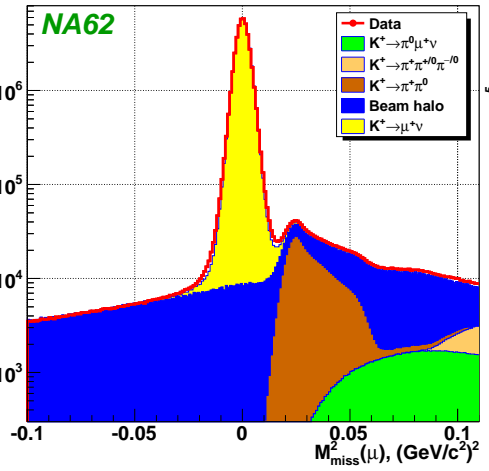

(c)

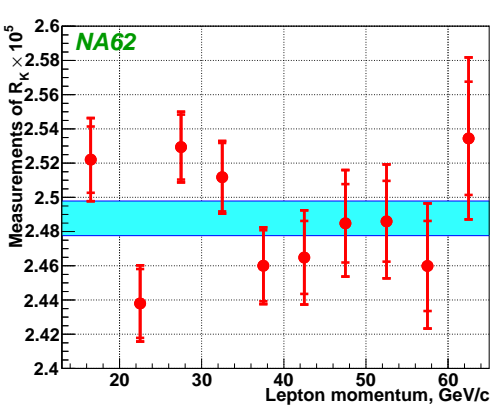

Figure 1: (a) Missing mass distribution and background contributions for $K_{e 2}(b)$ Missing mass distribution and background contributions for $K_{\mu 2}(c) R_{K}$ as function of lepton momentum

Table 1: Summary of uncertainties on $R_{K}\left(\delta R_{K} \cdot 10^{5}\right)$

\begin{tabular}{|llll|}
\hline Systematic & & & \\
\hline$K_{\mu 2}$ background & 0.004 & $K^{+} \pm \rightarrow e^{+} \gamma \nu\left(S D^{+}\right)$background & 0.002 \\
$K^{+} \rightarrow \pi^{0} e^{+} v, K^{+} \rightarrow \pi^{+} \pi^{0}$ backgrounds & 0.003 & Muon halo background & 0.002 \\
Spectrometer material composition & 0.002 & Acceptance correction & 0.002 \\
Spectrometer alignment & 0.001 & Electron id inefficiency & 0.001 \\
1-track trigger inefficiency & 0.001 & LKr readout inefficiency & 0.001 \\
\hline Total systematic & 0.007 & & \\
Total statistical & 0.007 & & \\
Total & 0.010 & & \\
\hline
\end{tabular}

Table 1 lists the main sources of uncertainty. This is the most precise measurement of $R_{K}$. The value measured by NA62 is consistent with the SM expectation and can be used to constrain several models of physics beyond the SM.

\section{Form factors in semileptonic decays}

Data for this analysis were collected in 2004 in a dedicated run with a special trigger setup requiring 1 track in the spectrometer and a minimum energy of $10 \mathrm{GeV}$ in the LKr calorimeter. Electron candidates should have a minimum momentum of $5 \mathrm{GeV} / c$, while muons are required to have $p>10 \mathrm{GeV} / c$ for the proper efficiency of the muon veto system. Particle identification is performed with cuts on $E / p$, where $\mathrm{E}$ is the energy in the calorimeter and $\mathrm{p}$ the momentum in the spectrometer: $E / p<0.2$ identifies a muon, while $0.95<E / p<1.05$ selects an electron. Events are rejected if there is extra energy in the calorimeter not associated with the track and with a cut on the missing mass under the $\mathrm{K}$ hypothesis $m_{m i s s}^{2}<0.01\left(\mathrm{GeV} / c^{2}\right)^{2}$. Background in the $K_{e 3}$ sample from $\pi^{+} \pi^{0}$ decays is reduced by a cut on the event transverse momentum to less than $0.1 \%$ with a $3 \%$ loss of the signal. For $K_{\mu 3}$ decays, background from $\pi^{+} \pi^{0}$ with $\pi^{+}$decaying in flight is suppressed by a two dimensional cut on $m_{\pi^{+}} \pi^{0}$ and on the $\pi^{0}$ transverse momentum. The 

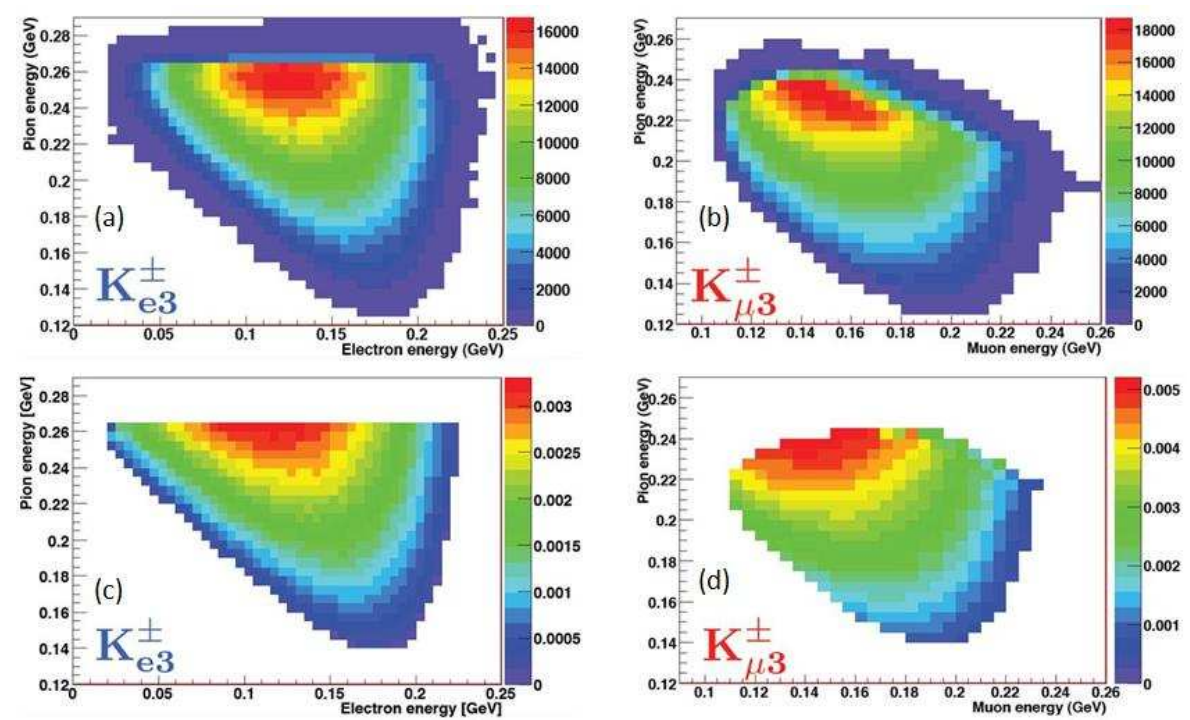

Figure 2: Reconstructed Dalitz plots for $K_{e 2}(a)$ and $K_{\mu 2}(b)$. Corrected Dalitz plots for $K_{e 2}(c)$ and $K_{\mu 2}(d)$ contamination is reduced to $0.5 \%$, but with a $24 \%$ loss of signal. Background from $\pi^{+} \pi^{0} \pi^{0}$ is estimated to be about $0.1 \%$ and no cut is applied. The total number of accepted decays is $4 \cdot 10^{6}$ $K_{e 3}^{ \pm}$and $2.5 \cdot 10^{6} K_{\mu 3}^{ \pm}$.

To extract the form factors, two dimensional fits to the Dalitz plot densities

$$
\rho\left(E_{l}^{*}, E_{\pi}^{*}\right)=\frac{d^{2} N\left(E_{l}^{*}, E_{\pi}^{*}\right)}{d E_{l}^{*} d E_{\pi}^{*}} \approx A f_{+}^{2}(t)+B f_{+}(t)\left(f_{0}(t)-f_{+}(t)\right) \frac{m_{K}^{2}-m_{\pi}^{2}}{t}+C\left[\left(f_{0}(t)-f_{+}(t)\right) \frac{m_{K}^{2}-m_{\pi}^{2}}{t}\right]^{2}
$$

are performed, with $\mathrm{A}, \mathrm{B}$ and $\mathrm{C}$ known functions of the decay kinematics. Instead of using the average kaon energy, the energies in the kaon rest frame are computed reconstructing the kaon energy of each event assuming no transverse $P_{t}$ of the kaon. With this definition, the energy resolution in the Dalitz plot is improved. The Dalitz plots obtained are shown in Figure 2 (a) and (b). The plots are divided in $5 \mathrm{MeV}^{*} 5 \mathrm{MeV}$ cells. Background, acceptance and radiative effects corrections are applied. For the radiative corrections, a special Montecarlo generator developed by KLOE [13] has been used. It has been verified that it is in agreement with a recent ChPT calculation[14]. Cells outside physical regions (due to resolution effects) are not used in the fit. The final corrected Dalitz plots are shown in Figure 2 (c) and (d).

Table 2: Preliminary results for quadratic and pole parametrization. $\lambda$ parameters are in unit of $10^{-3} \cdot M_{V}$ and $M_{S}$ are in $\mathrm{MeV} / \mathrm{c}^{2}$.

\begin{tabular}{|c|c|c|c|c|c|}
\hline & $\lambda_{+}^{\prime}$ & $\lambda_{+}^{\prime \prime}$ & $\lambda_{0}$ & $M_{V}$ & $M_{S}$ \\
\hline$K_{\mu 3}^{ \pm}$ & $26.3 \pm 3.0 \pm 2.2$ & $1.2 \pm 1.1 \pm 1.1$ & $15.7 \pm 1.4 \pm 1.0$ & $873 \pm 8 \pm 9$ & $1183 \pm 31 \pm 16$ \\
$K_{e 3}^{ \pm}$ & $27.2 \pm 0.7 \pm 1.1$ & $0.7 \pm 0.3 \pm 0.4$ & & $879 \pm 3 \pm 7$ & \\
Combined & $27.0 \pm 1.1$ & $0.8 \pm 0.5$ & $16.2 \pm 1.0$ & $877 \pm 6$ & $1176 \pm 31$ \\
\hline
\end{tabular}

The preliminary results for the quadratic and pole parametrization are listed in Table 2. In Fig. 3 the combined results reported by recent experiments are shown, with the $68 \%$ confidence level 

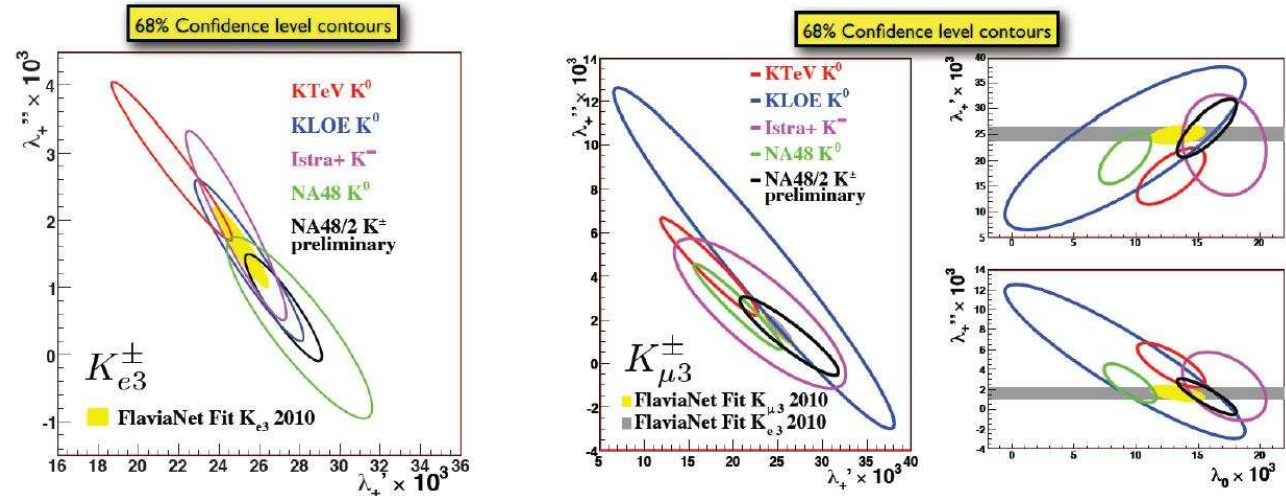

Figure 3: Preliminary combined quadratic fit results for $K_{l 3}$. The ellipses are $68 \%$ confidence level contours.

contours displayed for both neutral (KLOE, $\mathrm{KTeV}$ and NA48) and charged decays ( $\mathrm{K}^{-}$only in the case of ISTRA). The preliminary NA48 results shown here are the first high precision measurements obtained with both $K^{+}$and $K^{-}$mesons. The NA48 measurements are in good agreement with those of the other experiments (excluding $K_{e 3}^{0}$ from NA48 [15]) and compatible with the combined fit done by FlaviaNet [10].

\section{References}

[1] V. Cirigliano and I. Rosell, Phys. Rev. Lett. 99 (2007) 231801.

[2] A. Masiero, P. Paradisi and R. Petronzio, Phys. Rev. D74 (2006) 011701.

[3] A. Masiero, P. Paradisi and R. Petronzio, JHEP 0811 (2008) 042.

[4] A.G. Clark et al., Phys. Rev. Lett. 29 (1972) 1274.

[5] K.S. Heard et al., Phys. Lett. B55 (1975) 327.

[6] J. Heintze et al., Phys. Lett. B60 (1976) 302.

[7] F. Ambrosino et al., Eur. Phys. J. C64 (2009) 627; ibid. C65 )(2010) 703.

[8] C. Lazzeroni et al., Phys. Lett. B698 (2011) 105.

[9] C. Lazzeroni et al., Phys. Lett. B719 (2013) 326.

[10] M. Antonelli et al., Eur. Phys. J. C69 (2010) 399.

[11] J. R. Batley et al., Eur. Phys. J. C52 (2007) 875.

[12] V. Fanti et al., Nucl. Inst. Methods A574 (2007) 433.

[13] C. Gatti, Eur. Phys. J. C45 (2006) 417.

[14] V. Cirigliano, M. Giannotti and H. Neufeld, JHEP 11 (2008) 006.

[15] A. Lai et al., Phys. Lett. B647 (2007) 341. 